\title{
El concepto de 'valor' en Estados Unidos y en Colombia: análisis comparado de su contenido*
}

\section{Daniel Molano Camacho**}

RESUMEN. El presente artículo busca analizar el concepto de 'valor' desde una perspectiva de derecho comparado. Con dicho objetivo, el análisis propone una evaluación de la regulación estadounidense sobre el concepto de 'valor', así como un estudio de la misma idea en el derecho colombiano, todo con miras a analizar los posibles aprendizajes que, desde la experiencia extranjera pueden hacerse en la regulación colombiana en esta materia.

PALABRAS ClAVE: mercado de valores, security, valor, derecho bursátil, mercado de capitales.

\section{The Notion of Securities in the U.S. and Colombia:}

\section{A Comparative Assessment}

AbStRAct. This article aims to assess the securities notion from a comparative law point of view. It proposes an assessment over the U. S. securities regulation, as well as an evaluation of such regulation within the Colombian rules. With that aim, the

* Fecha de recepción: 9 de septiembre de 2019. Fecha de aceptación: 30 de marzo de 2020.

Para citar el artículo: Molano CAMACHO, D., "El concepto de 'valor' en Estados Unidos y en Colombia: análisis comparado de su contenido", Revista de Derecho Privado, n. ${ }^{\circ} 39$, julio-diciembre 2020 , 323-355, DOI: https://doi.org/10.18601/01234366.n39.13.

** Muñoz Tamayo \& Asociados, Bogotá, Colombia; abogado asociado. Universidad Externado de Colombia, Bogotá, Colombia; asistente de investigación en Derecho Procesal. Especialista en Derecho Financiero y Bursátil y Abogado, Universidad Externado de Colombia, Bogotá, Colombia. Contacto: daniel.molano@uexternado.edu.co. Orcid: 0000-0002-3259-8030. 
article seeks to identify the possible lessons to learn from the foreign securities regulations, specifically, within the Colombian securities market regulation.

KEYWORDS: securities markets, securities, capital markets, securities law.

Sumario: Introducción. i. Noción de 'valor' en Colombia. II. Noción de 'valor' en Estados Unidos. III. Aportes de la regulación de valores en Estados Unidos al régimen jurídico colombiano. Conclusiones. Referencias.

\section{Introducción}

El punto de partida de todo régimen jurídico, cualquiera que sea, desde un punto de vista teleológico y práctico, la mayor de las veces, descansa sobre su objeto. El régimen jurídico del mercado de valores no es la excepción, y así como se presenta como una subdivisión del derecho económico, cuyo objeto se orienta al estudio del mercado, en específico, poniendo énfasis en la circulación de recursos de capital ${ }^{1}$ entre los individuos ${ }^{2}$, el mercado de valores hace lo propio con el concepto de 'valor'.

Efectivamente, expresándose como corolario de la finalidad antedicha del derecho económico, el régimen del mercado de valores desarrolla esta especialidad jurídica a través de un concepto muy particular, cual es el de 'valor'. La pregunta inmediata, que como consecuencia evidente de tal afirmación se extrae, se orienta a interrogarse: ¿qué es un valor?

Tal concepto, que desde ya se anuncia no aparece jamás exento de problemáticas de todo orden -doctrinarias, dogmáticas, prácticas, etc.-, es el que se busca estudiar en esta oportunidad. El análisis propuesto busca ir sobre el concepto de 'valor' en Colombia, en específico, desde su intelección normativa a partir de la regulación vigente para tales propósitos. Así, el análisis de la noción acuñada, abordada a partir de sus antecedentes, elementos y restricciones contemporáneas, se enriquecerá, en términos propositivos, a través del análisis de su equivalente estadounidense, en particular para la confrontación de nuevas realidades de mercado que, aunado a los avances tecnológicos propios de nuestro siglo, enriquecen esa noción de security ${ }^{3} \mathrm{o}$ 'valor' en la actualidad.

En concreto, el presente estudio busca identificar el contenido predicable del concepto de 'valor' en Colombia y en Estados Unidos, para reconocer las similitudes y

1 Varón Palomino, J. C., Abella Abondano, G. D., Régimen del mercado de valores. Introducción al derecho del mercado de valores, tomo I, Bogotá, Ediciones Uniandes-Temis, 2017, 77.

2 Léase personas naturales o jurídicas.

3 En adelante, la referencia al concepto de valor en el derecho estadounidense se hará, sin perjuicio de ser utilizada bajo el sustantivo equivalente en castellano, a través del vocablo singular security, o por medio de su equivalente en plural, securities. 
las diferencias que, en uno u otro caso, puedan servir para enriquecer este concepto en Colombia desde un punto de vista práctico. Particularmente, se busca identificar la forma en que se lleva a cabo el análisis de dichos instrumentos en una y otra jurisdicción, para que, por medio del reconocimiento de puntos comunes, se pueda enriquecer la práctica jurídica nacional a través: del reconocimiento de nuevos instrumentos financieros como valores; de la posibilidad de exclusión de valores en determinados contextos; y de la asunción de nuevos matices en el papel de las autoridades que supervisan esta práctica.

Así, el artículo buscará, con la metodología de la equivalencia funcional ${ }^{4}$, analizar los conceptos mencionados, concretamente, de cara al análisis de los nuevos retos que hoy afrontan los mercados de valores desde una perspectiva teórica y práctica.

Para ello, el presente estudio evaluará: el concepto de 'valor' en Colombia; la noción de security en Estados Unidos; los aportes de la regulación estadounidense a la práctica nacional; y unas conclusiones.

\section{Noción de 'valor' en Colombia}

La noción de 'valor' en Colombia, así como en otras latitudes, desde el punto de vista conceptual, es el punto de partida para identificar derechos que, desde la perspectiva jurídica, dotan a operaciones financieras y de mercado de aquellas características jurídicas que hacen predicables de ellas una serie de facultades y obligaciones a las cuales tienen que ajustarse los agentes del mercado que intervienen en la dinámica del mercado de valores. De ahí la importancia práctica del concepto: la aplicación de unas reglas de juego específicas para los sujetos que participan en las operaciones a través de instrumentos que, conceptualmente, pueden tenerse como valor ${ }^{5}$.

\section{A. Antecedentes}

En Colombia, la normativa actual, establecida por la Ley 964 de 2005, encuentra una serie de antecedentes que, en su conjunto, han servido a la construcción del concepto de 'valor' que hoy se reconoce y aplica en el derecho del mercado de valores colombiano.

4 La metodología de la equivalencia funcional, propia del estudio comparado de figuras jurídicas, permite identificar "en qué casos un término del ordenamiento jurídico de la lengua de llegada puede ser utilizado como equivalente de traducción de un término jurídico de la lengua de partida”. HoLL, I., "Técnicas para la traducción jurídica: revisión de diferentes propuestas, últimas tendencias", Hermeneus: Revista de la Facultad de Traducción e Interpretación de Soria, Universidad de Valladolid, n. ${ }^{\circ} 14,2012,191-216$.

5 Esta afirmación se deriva, entre otras, de la lectura de los artículos 1 y 2 de la Ley 964 de 2005, que establece como marco de aplicación de la ley, y así también de las normas que la reglamenten o modifiquen, la captación de recursos del público a través de los instrumentos financieros denominados valores. 
La importancia de su reconocimiento descansa en la necesidad de identificar, desde el punto de vista normativo, cómo ha evolucionado la regulación relacionada con tal concepto y bajo qué realidades lo ha hecho, para con ello conjurar ciertas limitantes que de tales enunciados se fueron revelando.

Aun cuando gran parte de la normativa que antecede a la regulación actual no definió el concepto de 'valor' de manera concreta, la existencia de tales disposiciones constituye el antecedente obligado al régimen que desde principios de siglo se viene perfilando. Por ello, y dejando para un futuro el estudio profundo de tales antecedentes, el presente documento analizará los aspectos más relevantes de tal regulación.

\section{Decreto 2969 de 1960}

Sin duda, el primer antecedente en el régimen del mercado de valores, aunque no de concepto de 'valor' exactamente, es el Decreto 2969 de $1960^{[6]}$, que, en términos generales, fijó las reglas del funcionamiento de tal mercado.

La normativa, además de establecer el primer derrotero en materia de regulación bursátil, por lo menos, de manera estructurada, no definió de forma específica qué envolvía el concepto de 'valor'. Sin embargo, su artículo 11 listó, quizá precavidamente, algunos valores, entre ellos: (1) bonos y obligaciones emitidas por entidades de derecho público, establecimientos oficiales o semioficiales; (2) bonos y obligaciones emitidos por gobiernos extranjeros; (3) acciones y bonos emitidos por sociedades comerciales; (4) acciones, títulos de participación, cédulas hipotecarias, bonos de garantía general y de garantía específica, y otras obligaciones de establecimientos de crédito; (5) pagarés y letras de cambios garantizados por establecimientos bancarios; (6) metales amonedados en barras y divisas extranjeras; y (7) los demás valores de similar naturaleza a los expresados, en ese entonces, previa autorización de la autoridad de supervisión financiera.

Así, lo que en principio fue una regulación restringida al establecimiento de reglas de funcionamiento del mercado de valores sirvió por su parte para marcar un derrotero inicial de lo que, décadas más tarde, se desarrollaría con detalle en la Ley 964 de 2005.

\section{Ley 32 de 1979[7]}

Pocos años después de la expedición del Decreto 2969 de 1960, y en parte gracias al desarrollo que con el tiempo fue adquiriendo el mercado público de valores, se

6 Se trata del Decreto Ordinario 2969 del 26 de diciembre de 1960, expedido por la Presidencia de la República, mediante el cual se regularon las bolsas de valores en el país.

7 Aunque esta ley se enfoca primordialmente en desarrollar el concepto de oferta pública, debe tenerse como antecedente al concepto de valor, particularmente porque su redacción permite identificar algunas características de ellos. 
expidió la Ley 32 de 1979, por medio de la cual, entre otras, se creó la Comisión Nacional de Valores.

Dentro de esta regulación, el artículo $6 .^{\circ}$ estableció un primer parámetro para la consideración de 'valor' en la regulación colombiana, cuando definiendo la conformación del mercado público de valores dijo que éste estaría conformado por las operaciones sobre "documentos emitidos en serie o en masa, respecto de los cuales se realice oferta pública, que otorguen a sus titulares derechos de crédito, de participación y de tradición o representativos de mercancías".

De esta forma, el legislador colombiano establecía, tímidamente, algunos criterios para determinar cuáles eran las características de un valor, o por lo menos, de un instrumento negociado en el mercado de valores. De tal suerte, en Colombia se hacía un acercamiento al concepto de 'valor' a finales de la década de los setenta diciendo que serían tales aquellos documentos (1) emitidos en serie o en masa ${ }^{8}$; (2) objeto de oferta pública; y (3) concedentes a sus titulares de derechos de crédito, participación y de tradición o representativos de mercaderías.

Tal división tripartita de sus elementos, hoy perenne en algunos aspectos de la Ley 964 de 2005, sirvió de principal referencia en el mercado de valores sobre un tema que, hasta el momento, parecía generar cierta precaución por parte del legislador.

\section{Ley 35 de 1993}

La importancia de la Ley 35 de 1993, dada más por la realidad que revela que por la regulación misma que de 'valor' incorpora, se verifica en el hecho de que, de antaño, el legislador colombiano ha estructurado la regulación del mercado de valores sobre tal idea, inclusive cuando el concepto aún se encontraba en su más prístino desarrollo.

En tal ejercicio, por medio de la Ley 35 de 1993, el legislador colombiano se empeñó en establecer toda una serie de parámetros para llevar a cabo la labor de intervención que por conducto de la Constitución le corresponde a la rama ejecutiva del poder público ${ }^{9}$. En ese sentido, el Congreso de la República, que por su parte también cuenta con amplias facultades sobre este particular ${ }^{10}$, fijó las principales reglas bajo las cuales debía realizarse la actividad de intervención, haciendo énfasis, por supuesto, en la inspección, vigilancia y control.

8 Recuerda Fagua que bajo esa normativa en particular no se realizaba ninguna distinción entre la noción de documentos y la de valores, utilizándose ambos conceptos de manera indistinta a lo largo de la regulación. Varón Palomino, J. C., Abella Abondano, G. D., Régimen del mercado de valores. cit., n. ${ }^{\circ} 1,231$.

9 En ese sentido el numeral 24 del artículo 189 de la Constitución Política colombiana establece como deberes del presidente de la República: "Ejercer, de acuerdo con la ley, la inspección, vigilancia y control sobre las personas que realicen actividades financiera, bursátil, aseguradora y cualquier otra relacionada con el manejo, aprovechamiento o inversión de recursos captados del público. Así mismo, sobre las entidades cooperativas y las sociedades mercantiles".

10 Constitución Política colombiana, lit. d, num. 19, art. 150. 
De manera que, aunque parecería percibirse como innecesaria la reproducción de esta norma, lo cierto es que no puede desconocerse que su relevancia es toda, no solo porque establece los criterios fundamentales que deben guiar la actividad de intervención mencionada, sino también porque el objeto de dicha actividad, en buena parte de los casos, gira en no pocas ocasiones sobre el concepto de 'valor' al que este artículo se refiere.

\section{Resoluciones 645 del 14 de agosto de 1992; 1394 del 4 de noviembre de 1993; y 400 del 22 de mayo de 1995 de la Superintendencia de Valores}

A todos los antecedentes mencionados hasta el momento se sumaron las regulaciones que, producto de las facultades derivadas del Decreto Constitucional 2739 de $1991^{[11]}$, expidió la Superintendencia de Valores.

Dicha regulación, a su vez, contribuyó a la delimitación del concepto de 'valor', pues cada una de las resoluciones enunciadas fijó un antecedente a lo que hoy en día regula la Ley 964 de 2005 a propósito de este concepto.

La Resolución 645 de 1992, en particular, reguló la inscripción de nuevos valores en el Registro Nacional de Valores, para lo cual hizo un valioso esfuerzo en la precisión de figuras tales como los títulos de participación y contenido crediticio; las unidades de participación en fondos especiales constituidos a través de negocios fiduciarios; entre otros que, a más de contribuir a la construcción del mecanismo de titularización en Colombia, empezaron a delinear el concepto con que hoy se cuenta sobre el particular.

Esta norma fue derogada por la Resolución 1394 de 1993, que al regular el mecanismo de titularización en Colombia, incluyó en su contenido la idea de "nuevos instrumentos financieros" 12 , que, producto de los procesos de titularización, se mostraron como antecedente indudable de la noción que se tiene hoy en día en el artículo 5.6.1.1.5 del Decreto 2555 de 2010, y por supuesto, de 'valor'.

Finalmente, la Resolución 400 de 1995 entró al panorama a cumplir un papel relevante, esencialmente porque con ella se regularon y actualizaron las normas expedidas por la Sala General de la Superintendencia de Valores, y, en particular, se compilaron las nociones explicadas con anterioridad.

\section{El Decreto 1168 de 1993}

Un último antecedente que puede reconocerse, en lo que toca con la idea de 'valor' en Colombia, es el contenido en el Decreto Reglamentario 1168 de junio 24 de 1993.

11 El Decreto 2739 de 1991, aunque no regula concretamente la naturaleza del concepto de valor, establece los parámetros para que la antigua Comisión Nacional de Valores pasare a ser Superintendencia de Valores. Esta transformación fue la que permitió, entre otras, que se expidieran las resoluciones mencionadas en el presente acápite.

12 Superintendencia de Valores, Resolución 1394 de 1993, art. 5. 
En dicha norma se incluyó la noción de documentos emitidos en serie y en masa que, sin duda, constituye un antecedente al concepto estudiado. De acuerdo con el texto de la norma, tendrán dicha calidad aquellos documentos que: (1) se creen en acto único o actos sucesivos que den lugar, por lo menos, a 20 títulos; (2) tengan un contenido sustancial homogéneo (patrón común); (3) gocen de igual naturaleza jurídica; (4) cuenten con la misma ley de circulación; (5) provengan de un mismo emisor; y (6) tengan vocación circulatoria ${ }^{13}$.

\section{B. Noción actual de 'valor' en Colombia}

Establecidos los principales antecedentes en materia de regulación del concepto de 'valor', creemos pertinente ir sobre el concepto que, regulado en el artículo 2. de la Ley 964 de 2005, sirve hoy como punto de partida para el estudio del régimen del mercado de valores colombiano.

\section{Ley 964 de 2005}

La Ley 964 de 2005, a nuestro juicio la primera regulación integral del mercado de valores colombiano, incorporó la definición concreta de lo que ha de considerarse valor dentro del contexto nacional.

Sobre el particular, y conscientes de la rica producción bibliográfica que ha sido desarrollada recientemente en el contexto colombiano ${ }^{14}$ sobre este aspecto, la referencia será breve, y se orientará a la explicación general del valor y sus elementos.

Dicho lo anterior, lo primero que corresponde hacer es recordar la definición incluida en el artículo 2 de la Ley 964 de 2005, según el cual, será valor: “[...] todo derecho de naturaleza negociable que haga parte de una emisión, cuando tenga por objeto o efecto la captación de recursos del público [...]”".

La definición, luego de la cual se enuncia toda una serie de documentos que, desde un principio, se tienen como valores, estructura la noción de 'valor' a partir de tres requisitos en particular, esto es: (1) que sean derechos de naturaleza negociable; (2) que hagan parte de una emisión; y (3) que tengan por objeto o efecto la captación de recursos del público.

13 Decreto 1168 de 1993, art. 1.

14 Véase, entre otros: López Roca, L. F., Baquero Herrera, M. H. y Corredor Higuera, J. A. (eds.), Estudios jurídicos sobre el mercado de valores, Universidad Externado de Colombia, Bogotá, 2018, 799; Blanco Barón, C. y Baquero Herrera, M. (eds.), Cinco años de la Ley 964 de 2005: ¿se están cumpliendo sus objetivos?, Bogotá, Universidad Externado de Colombia, 2011, 157-181; CorreDOR, J. A., “Trece años de vigencia de la Ley 964 de 2005: análisis de sus efectos en el mercado de valores y puntos para tener en cuenta para futuras modificaciones", Revista Prolegómenos, Editorial Neogranadina, vol. 22 (43), 2019,21-41; además del trabajo realizado sobre el mismo particular por la Universidad de los Andes, cuya obra se cita a lo largo del presente estudio. 
Ahora: si bien es cierto que hay quienes en la intelección de la norma reconocen un cuarto elemento ${ }^{15}$, creemos que, para efectos del presente estudio, la identificación de los tres elementos que de la mera lectura de la norma saltan a la vista es más que suficiente.

\section{Elementos de la definición}

Reconocidos los elementos como se indicó con anterioridad, corresponde ahora establecer el significado que de cada uno de ellos se deriva. Sin embargo, debido a la extensión y el objetivo del presente estudio, y así también al reconocimiento de suficiente doctrina sobre el particular ${ }^{16}$, habremos de hacer una simple ilustración general de los elementos que lo componen, toda vez que, si bien es necesario realizar su identificación para efectos de llevar a cabo la evaluación de existencia de un valor, lo cierto es que su consideración resulta importante para el presente artículo, en la medida en que permita mostrar cuándo se está frente a un valor en el derecho colombiano, para con ello poder contrastar esto con la regulación de security en el derecho estadounidense.

\section{a. Derechos de naturaleza negociable}

El primero de los elementos para la identificación de 'valor' en el régimen jurídico colombiano -la corroboración de un derecho de naturaleza negociable- obliga a ir sobre un particular de suma importancia, que responde a la pregunta ¿Qué derechos son negociables bajo la regulación de valores en Colombia?

15 Fagua, a propósito de la identificación de los elementos del concepto de valor bajo la norma, reconoce que tales son: "(1) un derecho instrumentado en un documento, físico o electrónico; (2) ser negociable; (3) hacer parte de una emisión, y (4) tener por objeto o efecto la captación de recursos del público". Sin embargo, creemos que el adjetivo verbal "negociable", en el contexto de la norma, es utilizado para calificar aquellos derechos que pueden ser entendidos como valor, y no como un elemento adicional de la definición. Así mismo, consideramos que la instrumentalización de tales derechos a través de un documento físico o electrónico es una conclusión que se advierte de la lectura concordante y complementaria de otras normas que hacen parte de régimen del mercado de valores ( $v$. $g r$., artículos 4 y 5 de la Ley 27 de 1990), mas no de la lectura exclusiva del enunciado normativo contenido en el artículo 2 de la Ley 964 de 2005. Entonces, aunque reconocemos la indudable necesidad de que tales derechos estén incorporados en un documento, lo cierto es que tal aspecto, en principio, no debe ser considerado un elemento que haga parte de la definición legal, sin perjuicio claro, que en desarrollo y lectura de las normas que complementan a tal ley marco en materia del mercado de valores, se tenga presente la necesidad de incorporarlo como tal. Así, aun cuando entendemos que la posible verificación de este elemento se deba a la referencia que por medio del parágrafo $4^{\circ}$ del artículo 2 de la Ley 964 de 2002 se hace al régimen de títulos valores, consideramos que el texto normativo de la definición se restringe al inciso primero del mismo, y no a las demás disposiciones que entran a enriquecer ese concepto claramente definido. VARÓN PALOMINo, J. C. y Abella Abondano, G. D., Régimen del mercado de valores, cit. en n. ${ }^{\circ}$ 1, 233-235.

16 Véase, entre otras, las mencionadas en la nota 15. 
Sobre lo anterior, encontramos ilustrativas las reflexiones de Varón Palomino y Abella Abondano, quienes coincidiendo sobre la composición tripartita de elementos del concepto, analizan este primer punto desde la evaluación del contenido patrimonial, la transmisibilidad, la fungibilidad y la representación del respectivo derecho ${ }^{17}$.

Así, sobre el primero de los predicados, esto es, frente al contenido patrimonial del valor, las consideraciones han sido claras, en el sentido de que, inclusive desde la Ley 32 de 1979, la intelección de tal aspecto en particular se ha dado, por un lado, desde el contenido propio que les ha sido atribuido a los títulos-valores, esto es, crediticios, de participación, de tradición o representativos de mercaderías ${ }^{18}$, y por el otro, desde las incorporaciones que recogió después el Decreto 2555 de 2010, las cuales, por supuesto, se ampliaron extensamente bajo la regulación vigente.

En esta última normativa, los valores pueden, bajo la noción de "nuevos instrumentos financieros", adquirir una naturaleza mixta, conforme a la cual, estos pueden también incluir, además de los derechos que confiere el título de participación, la previsión de amortización, una rentabilidad, o un límite máximo de participación ${ }^{19}$.

De esta suerte, las previsiones relativas a la patrimonialidad mencionadas hoy en día son ampliadas extensamente por la nueva regulación, bajo la cual se admiten nuevos derechos de naturaleza económica, abriendo, a nuestro juicio de forma saludable, el espectro que frente a nuevas realidades puede llegar a tener que afrontar el mercado de valores.

Por su parte, y concerniente a la transmisibilidad, se ha dicho que el vocablo relativo a la negociabilidad refiere a la vocación que tiene el respectivo valor de ser transmitido o negociado en el mercado de dichos instrumentos, sin consideración especial a su ley de circulación, esto es, aquellas propias del endoso, el mecanismo de anotación en cuenta ${ }^{20}$, o de cualquier otro que cumpla idénticos objetivos.

A lo anterior se suma, por otro lado, la fungibilidad del respectivo derecho. Tal característica refiere a la necesidad que el derecho, en tanto homogéneo, pueda ser reemplazado por otra prerrogativa económica que haga parte de la misma emisión ${ }^{21}$. Ello implica, así mismo, que el derecho integrante de una misma emisión deba gozar de un idéntico contenido y ley de circulación ${ }^{22}$.

A todo ello, y como estadio final del estudio del primer elemento de la noción de 'valor', se suma la representación del derecho como parte integrante de este. Sobre

17 Varón Palomino, J. C., Abella Abondano, G. D., Derechos fiduciarios y mercado de valores: reflexiones frente a la normativa colombiana, Ediciones Uniandes-Asociación de Fiduciarias, Bogotá, 2013, 39-40.

18 Código de Comercio colombiano, art. 619.

19 En ese sentido, el numeral 3 del artículo 5.6.1.1.5 del Decreto 2555 de 2010 estableció: “[...] Son aquellos que de manera adicional a los derechos que confiere un título de participación pueden ser amortizables o pueden tener una rentabilidad mínima o un límite máximo de participación".

20 Varón Palomino, J. C., Abella Abondano, G. D., Régimen del mercado de valores, cit. en n. .o 18, 44.

21 Ibid., 44 y 45.

22 Ibid., 45. 
ello, la doctrina ha recordado un particular que, hoy más que evidente, sobresale en la normativa colombiana, cual es, en este caso, la ausencia de disposición expresa sobre el asunto 23 .

Sobre ello, aunque creemos que la evaluación no debe ser muy extensa, consideramos que la ausencia de una disposición específica en la materia se debe, más allá de una laguna o un descuido en la regulación, a una realidad que no puede pasarse por alto en el mercado de valores, cual es su dinámica y cambio constante. En ese sentido, creemos que la ausencia de una disposición expresa sobre ese particular, dentro del ordenamiento jurídico colombiano, es más que afortunada, no solo porque escapa al positivismo estricto que en muchas ocasiones ha caracterizado al régimen colombiano, sino también porque abre las puertas para la asimilación de los fenómenos que la práctica misma del mercado de valores exige.

\section{b. Que hagan parte de una emisión}

El segundo de los elementos obliga a ir sobre otros conceptos que escapan de la restrictiva lectura literal de la norma. En este caso, el segundo componente de la definición hace imperativo analizar el concepto de 'emisión', y, por consiguiente, el de 'mercado de valores', pues, siendo éste el escenario natural de las emisiones, se debe tener claridad sobre su intelección.

En ese sentido, la definición de la Real Academia, como punto de partida, se muestra ilustrativa, en especial para definir las emisiones como la "acción de emitir", y por ello, como el "conjunto de títulos o valores, efectos públicos o efectos de comercio o bancarios, que de una vez se ponen en circulación" ${ }^{24}$. Con ello la definición da a entender que emisión es, en efecto, esa acción por medio de la cual se ponen a circular valores, en este caso, dentro de un mercado en particular, cual es el mercado de valores.

Ahora: desde el punto de vista jurídico, la definición de 'emisión' se echa de menos en el régimen jurídico colombiano. Por ello, nos valemos de los criterios para determinación de 'valor' establecidos por Fagua, quien, para dotar de contenido dicho concepto, recuerda que la emisión “[...] connota la articulación de los diferentes actos y requisitos jurídicos que se requieren para emitir y colocar en el mercado de valores" 25 . Así mismo, señala el autor que deberá tenerse en cuenta, dentro del concepto de "emisión de valores' "[...] que se trate de valores de la misma naturaleza, creados en virtud de un acto único por parte del órgano de la entidad emisora competente para ello y sobre la base de que se haya dado cumplimiento a las formalidades legales que la ley y los estatutos establezcan" ${ }^{26}$. 
Diferente lo anterior a la idea de oferta pública de valores ${ }^{27}$, que, como podrá deducirlo el lector, excede en alguna medida los alcances del presente estudio, en específico porque el universo de las emisiones públicas de valores, sus detalles y regulaciones abordan un espacio tan interesante como amplio, el cual, para efectos del presente análisis, no enriquece de particular forma la propuesta que se expone.

\section{c. Que tenga por objeto o como efecto la captación de recursos del público}

El tercero de los requisitos, en lo que tiene que ver con el reconocimiento de un valor en Colombia, guarda relación con un elemento que puede verificarse desde dos puntos de vista, esto es, el teleológico y el fáctico. Así, el reconocimiento de este tercer requerimiento se puede constatar en los eventos en que en el plano fáctico se verifique efectivamente la captación de recursos del público (efecto), en cuyo caso tendrán que analizarse los aspectos fácticos de la emisión; y, en aquellas circunstancias en las que se constante, la intención de captación (objeto).

Este elemento, a nuestro juicio, en principio no planea problema alguno, pues si verificado el cumplimiento de las normas del mercado de valores se establece la verdadera captación de recursos del público, en este caso por medio de la emisión de dichos derechos, se estará ante un verdadero valor, claro, siempre que se hubieren verificado los anteriores requisitos.

Con todo, debe precisarse que en uno y otro caso, esto es, bien sea como efecto o como objeto, para que se verifique la existencia de un valor, a la captación respectiva tienen que sumarse los dos requisitos mencionados con anterioridad, pues de lo contrario no se estará ante la captación estudiada en este documento, sino a ante una de diferente naturaleza.

\section{Sobre los valores no listados en el artículo $2 .^{\circ}$ de la Ley 964 de 2005}

La interpretación de la norma en comento plantea un problema que, situándose en el marco netamente práctico, resalta la importancia del presente estudio. En efecto, la necesidad de evaluar y de dotar determinados derechos o instrumentos de la calidad de valor plantea un problema práctico en cuanto obliga a preguntarse si, bajo la previsión del literal b del artículo 4. ${ }^{\circ}$ de la Ley 964 de 2005 debe haber un reconocimiento previo por parte del Gobierno Nacional de calidad de valor del instrumento o derecho respectivo.

27 El Decreto 2555 de 2010, en su artículo 6.1.1.1.1, definió la oferta pública de valores como aquella dirigida: (1) a más de 100 personas determinadas; o (2) a personas no determinadas. Con dicha definición incluyó 3 excepciones, relacionadas con ofertas: (1) de acciones y boceas dirigidas a menos de 500 accionistas de la emisora; (2) de acciones resultado de órdenes de capitalización; y (3) de acciones no inscritas en el RNVE y dirigidas a personas con contrato laboral vigente o miembros de la junta directiva de la sociedad. 
Sobre ello, nos adherimos a la posición de López Roca y Fagua Guauque, en tanto creemos que la calidad de valor de dichos instrumentos no listados en el artículo 2. ${ }^{\circ}$ de la Ley 964 de 2005 no deviene del reconocimiento de la rama ejecutiva del poder público, sino del cumplimiento de los requisitos contenidos en la ley. Consideramos, además, que en el terreno práctico la ausencia de dicho reconocimiento entorpece el empleo efectivo de dichos valores, pues lo que en teoría debería verificarse con facilidad en la práctica se echa de menos, pues pocos o ningún instrumento no listado en el artículo mencionado ha sido dotado de los efectos propios de un valor ${ }^{28}$.

Dicho reconocimiento, a más de los problemas prácticos señalados por la doctrina mencionada ${ }^{29}$, a la cual nos sumamos en su totalidad, creemos es un punto fundamental en el presente análisis, pues, como se señalará más adelante, la influencia estadounidense, que por demás creemos pesa cada día más en el régimen colombiano, sirve como un importante derrotero que tener en cuenta por quienes, a nuestro juicio equivocadamente, requieren de quien utiliza un instrumento no listado en la Ley 964 de 2005, una reglamentación gubernamental.

En ese sentido, la Superintendencia Financiera en reiteradas ocasiones ha mencionado que "[...] si bien el listado contenido en el artículo 2. ' de la Ley 964 de 2005 es enunciativo y no taxativo, para poder reconocer como valores, instrumentos distintos a los señalados en la mencionada disposición y que cumplen con los requisitos señalados en el primer inciso de la mencionada norma, se requiere de la correspondiente reglamentación gubernamental" 30 .

Todo lo cual, al final, y distanciándonos, como se advirtió desde el principio, de dicha interpretación ${ }^{31}$, nos obliga a preguntarnos si en realidad tal facultad, a nuestro

28 Sobre ello López Roca recuerda cómo instrumentos que antes de la redacción y expedición de la norma general del mercado de valores habrían carecido de la calidad de valores, hoy pueden valerse de los beneficios en tanto tales, por ejemplo, a través de la titularización y la utilización de mecanismos fiduciarios como el empleado en proyectos como el edificio Bacatá (edificación en la ciudad de Bogotá, en la que se emitieron derechos fiduciarios denominados FIDIS) y similares. López RocA, L. F., Baquero Herrera, M. H. y Corredor Higuera, J. A. (eds.), Estudios jurídicos sobre el mercado de valores, cit. en n. ${ }^{\circ} 15,178$.

29 Ibid., 176-178.

30 Esa ha sido la posición de la autoridad en reiteradas ocasiones. Véase, entre otros, el Concepto 2016054365-015 del 28 de julio de 2016 y el Concepto 20056-946 del 27 de julio de 2005 de la Superintendencia Financiera de Colombia.

31 En Colombia, la titularización ha permitido la emisión de títulos no tradicionales o nuevos títulos. Algunos de ellos son: (1) los títulos producto de la titularización inmobiliaria como los derivados del Patrimonio Autónomo Estrategias Inmobiliarias, en el cual se emitieron títulos participativos Estrategias Inmobiliarias (“TEIS"), y en el cual participó Corficolombiana como agente de manejo, Estrategias Corporativas como originador, Corredores Asociados S. A. (hoy Corredores Davivienda) como colocador y Brigard \& Urrutia como asesor legal; (2) los títulos de cartera hipotecaria atados a la unidad de valor real (TIPS UVR); (3) los TIPS en pesos; (4) los títulos de cartera inmobiliaria improductiva (ТЕСН); (5) los títulos de cartera de consumo o libranza (TIL); (6) los títulos de cartera de redescuento (TER); o (7) los FIDIS, emitidos para representar participaciones del edificio BD Bacatá. Sin embargo, aunque estos títulos son un avance indudable en el mercado de valores colombiano, su creación se encuentra indefectiblemente atada a la rigidez de la titularización, y sigue siendo insuficiente y rezagada si se compara con el mercado estadounidense. Véase el Prospecto de Colocación, 
juicio producto de una errónea lectura de la norma, realmente debería descansar en dicha autoridad, o si, por el contrario, como sucede en el caso estadounidense, debería obedecer a un proceso dentro del cual, pasando por el crisol del abogado asesor del emisor, y en caso de controversia por el del juez, se llegue a la verdadera determinación del valor, sin sacrificar, en el camino, la mecánica práctica con la que debe contar el mercado de valores para hacer rápida circulación de los instrumentos que se negocian en su seno.

\section{Noción de 'valor' en Estados Unidos}

El derecho estadounidense, pionero sin duda en el régimen jurídico del mercado de valores, se muestra hoy como un referente obligado para todo aquel que desee ir sobre la noción y la realidad que implica el concepto de 'valor' en todas sus dimensiones.

Ajustado a las realidades propias del derecho anglosajón, y teniendo presentes las diferencias que de ellas se desprenden ${ }^{32}$, este estudio pretende, en lo que podría denominarse un conocimiento inescapable para todo operador jurídico interesado en el mercado de valores, estudiar las particularidades propias del concepto de 'valor' en el derecho estadounidense.

Para ello se analizará el concepto de security que, desde el derecho estadounidense, presenta el punto de partida el régimen del mercado de valores angloamericano o, lo que es lo mismo, de lo que se denomina securities regulation ${ }^{33}$.

\section{A. Noción de security en el Securities Act \$2(a)(1) y en el Securities Exchange Act $\$ 3(a)(10)$}

El presente acápite buscará, desde la noción legal incluida en las normas correspondientes, evaluar la noción de security que se establece dentro de los estatutos estadounidenses. Para ello, el estudio irá sobre las dos normas fundamentales en materia del mercado de valores en Estados Unidos, esto es: la Securities Act de 1933 y la Securities Exchange Act de 1934, cuyo contenido, ya cercano a cumplir un siglo de vigencia, presenta el primer acercamiento de lo que se puede considerar un security en tal latitud.

del Programa de Emisión y Colocación de Títulos Participativos del Patrimonio Autónomo Estrategias Inmobiliarias de julio de 2013, disponible en [www.bvc.com.co/recursos/emisores/Prospectos/ Titulos_Participacion/Adenda_07_Prospecto_Estrategias_Inmobilarias_2013.pdf].

32 Para ahondar en las principales diferencias de la regulación financiera entre Estados Unidos y los regímenes del civil law, véase Alcaraz Varó, E.; Campos Pardillos, M. A. y Miguélez, C., El inglés

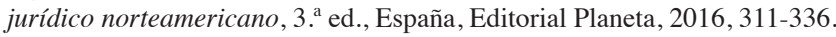

33 Para el desarrollo del presente estudio se tomará como referencia la clasificación general de securities realizada por Palmiter, a la cual, a más de las referencias directas y normativas que se le realizarán durante todo el texto, se anudarán las consideraciones propias de cada uno de los particulares y sus adicionales. Palmiter, A. R., Securities regulation, 7. . ed., New York, Wolters Kluwer, 2017, 49-79. 


\section{Instrumentos financieros taxativos (list of financial instruments)}

El régimen del mercado de valores estadounidense define, desde las dos leyes mencionadas, los securities desde una perspectiva enunciativa, bajo la cual se incluye una serie de instrumentos que se consideran security desde un primer momento. Dentro de tales instrumentos, muchos de los cuales coinciden en la Securities Act $\S 2(\mathrm{a})(1)^{34}$ y en la Securities Exchange Act $\$ 3(\mathrm{a})(10)^{35}$, se encuentran: las acciones, los bonos, los títulos de deuda, las notas, entre otros que, en tanto instrumentos financieros típicos del mercado de valores, a priori, son considerados tal.

Todas estas figuras, contenidas en la norma como instrumentos financieros a los que se les reconoce desde un primer momento la calidad de security ${ }^{36}$, encuentran, por un lado, un matiz a través de la "cláusula de contexto" o "context clause" ${ }^{37}$, bajo la cual, serán securities todos los documentos mencionados a menos que el contexto lo requiera de otro modo ("the context otherwise requires"), y por otro, un amplificador o "catchall", que jurisprudencialmente ha venido a calificar como securities determinados instrumentos que, a pesar de no ser considerados en la lista pretéritamente mencionada, son verdaderos valores.

34 Recuerda la norma la definición en los siguientes términos: "The term 'security' means any note, stock, treasury stock, security future, security-based swap, bond, debenture, evidence of indebtedness, certificate of interest or participation in any profit-sharing agreement, collateral-trust certificate, preorganization certificate or subscription, transferable share, investment contract, voting-trust certificate, certificate of deposit for a security, fractional undivided interest in oil, gas, or other mineral rights, any put, call, straddle, option, or privilege on any security, certificate of deposit, or group or index of securities (including any interest therein or based on the value thereof), or any put, call, straddle, option, or privilege entered into on a national securities exchange relating to foreign currency, or, in general, any interest or instrument commonly known as a 'security', or any certificate of interest or participation in, temporary or interim certificate for, receipt for, guarantee of, or warrant or right to subscribe to or purchase, any of the foregoing".

35 La norma define security así: "The term 'security' means any note, stock, treasury stock, security future, security-based swap, bond, debenture, certificate of interest or participation in any profitsharing agreement or in any oil, gas, or other mineral royalty or lease, any collateral-trust certificate, preorganization certificate or subscription, transferable share, investment contract, votingtrust certificate, certificate of deposit for a security, any put, call, straddle, option, or privilege on any security, certificate of deposit, or group or index of securities (including any interest therein or based on the value thereof), or any put, call, straddle, option, or privilege entered into on a national securities exchange relating to foreign currency, or in general, any instrument commonly known as a 'security'; or any certificate of interest or participation in, temporary or interim certificate for, receipt for, or warrant or right to subscribe to or purchase, any of the foregoing; but shall not include currency or any note, draft, bill of exchange, or banker's acceptance which has a maturity at the time of issuance of not exceeding nine months, exclusive of days of grace, or any renewal thereof the maturity of which is likewise limited".

36 Loss, L. y Seligman, J., Fundamentals of securities regulation, 5. a ed., Wolters Kluwer, 2004, 231.

37 Steinberg, M. I. y Kaulbach, W. E., "The Supreme Court and the Definition of Security: The Context Clause, Investment Contract Analysis, and Their Ramifications", en Vanderbilt Law Review, vol. 40, n. $^{\circ} 3,1987,504-512$. 


\section{Definición macro de security (catchall)}

Como se indicó, adicional a la lista enunciativa de securities, existe una noción amplia de ellos, según la cual: (1) existen securities no enunciados en la norma; y (2) aquellos que los están no son siempre valores.

Esta idea, cuyo desarrollo se presenta en el siguiente acápite, es de especial interés para el presente estudio, pues revela la adopción de una noción que, a pesar del ritualismo de la tradición romano-germánica ${ }^{38}$, puede enriquecer la noción nacional desde la comprensión de su equivalente funcional en Estados Unidos.

\section{B. Noción amplia de security (catchall)}

La noción amplia de security en Estados Unidos ha sido desarrollada por la jurisprudencia estadounidense a través de la implementación de dos tests concretos: (1) el investment contracts test y (2) el risk capital test ${ }^{39}$.

La inclusión de estos dos conceptos, como se debe anunciar, es fundamental en la evaluación de la existencia de un security, en especial porque ellos permiten establecer unos criterios claros para la identificación de un nuevo valor en Estados Unidos.

\section{Contratos de inversión (investment contracts) y el Howey test}

La noción de contratos de inversión o "investment contracts" encuentra como antecedente la decisión de la Corte Suprema de Justicia de los Estados Unidos que en SEC v. W. J. Howey Co., 328 U. S. 293 (1946) definió los contratos de inversión ${ }^{40}$ como transacciones en las que

[1] a person invests money, [2] in a common enterprise, and [3] is led to expect profits, [4] solely from the efforts of others ${ }^{41}$.

38 Merryman, J. H., The Civil Law Tradition: An Introduction to the Legal Systems of Europe and

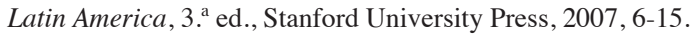

39 Este último test fue empleado por primera vez por la Corte Suprema de California en Silver Hills Country Club v. Sobieski, 55 Cal. 2d 811, 361 P.2d 906, 13 Cal. Rptr 186 (1961), cuando se reconoció la calidad de security a la membresía de un club social, a pesar de no haber una expectativa de ganancia como en el Howey test, bajo la lectura del California Corporate Securties Act. Este test, que inicialmente fue concebido en el ámbito estatal, luego fue adoptado en las cortes federales, por ejemplo, en Securities and Exchange Commission v. Latta, 250 F. Supp. 170, N. D. cal. 1965; Khadem v. Equity Securities Corp., 494 F.2d 1224, 9th Cir. 1974; o en Great Western Bank \& Trust v. Kotz, 532 F.2d 1252 9th Cir. 1976.

40 Se recuerda la Securities Act de 1933, §2(a)(1), que dice: “The term 'security' means any note [...], investment contract $[\ldots] "$.

41 En traducción propia: "[1] una persona invierte dinero, [2] en una empresa en común, y [3] espera recibir ganancias, [4] únicamente producto del esfuerzo de otros". 
Así, en la tradición estadounidense los requisitos para que se configure la corroboración de un security vienen dados por cuatro elementos, que según Palmiter ${ }^{42}$ se pueden calificar en cuatro grandes criterios, a saber: (1) investment, (2) commonality, (3) expected profits y (4) efforts of others ${ }^{43}$.

Así, el primero de los elementos que ha de verificarse, de acuerdo con lo dicho, es la necesidad de una inversión (investment) que, pudiendo ser expresada en dinero $\mathrm{u}$ otras especies, debe encaminarse a la producción de una ganancia o ingreso.

En segundo lugar, tiene que corroborarse una comunidad (commonality) en el esquema de inversión, en el sentido de que, cuando menos, debe haber una comunidad horizontal en la que múltiples inversionistas converjan en un esquema para la realización de intereses comunes. Ahora: si bien por lo general se exige un esquema horizontal como el mencionado, puede ocurrir también que en ocasiones se presente una vertical commonality, en la que los intereses de un solo inversor converjan con los de un único administrador ${ }^{44}$.

En tercer lugar, debe percibirse una expectativa de ganancias (expected profits) en la inversión. Esta, además, debe derivarse de: (1) las ganancias de la empresa o (2) réditos de la inversión reconocidos anticipadamente, y en ningún caso puede derivar de los simples aportes adicionales realizados por el inversionista ${ }^{45}$.

Finalmente, y con gran relación con dos de los problemas propios del derecho financiero, esto es, el de la teoría de la agencia y el de la acción colectiva ${ }^{46}$, se presenta el esfuerzo de otros (effort of others) como el elemento final del Howey test. Bajo este criterio se debe corroborar que los réditos o recursos que el inversionista perciba provengan del esfuerzo realizado por el administrador y no por el de los inversionistas mismos. Sin embargo, y a pesar de que esta es la regla general, en circunstancias excepcionales se ha reconocido que la ganancia provenga no ya del esfuerzo de un tercero, sino de aquella empleada por el mismo inversionista ${ }^{47}$.

Por su parte, la aplicación del concepto estudiado no se ha hecho esperar, y en muchas ocasiones se ha llevado a cabo en circunstancias inesperadas, algunas de las cuales se pasan a reseñar, en este caso, como ilustración de la aplicación concreta de este test.

El primer hecho que se puede relacionar, sin duda, es aquel del que surgió el Howey test. En este, la Corte Suprema de Justicia de Estados Unidos consideró que la venta de un cultivo de 48 árboles de citrón, por ser administrados, cultivados y

42 Palmiter, A. R., Securities regulation, $k .$, n. $^{\circ} 34,50$.

43 Dichos elementos, aunque sin la connotación que les es propia en la lengua inglesa, pueden traducirse al castellano como: (1) inversión; (2) comunidad; (3) expectativa de ganancia; y (4) esfuerzo de otros.

44 PALmiter, A. R., Securities regulation, cit., 50.

45 Idem.

46 Varón Palomino, J. C. y Abella Abondano, G. D., Régimen del mercado de valores, cit., n. ${ }^{\circ}$ 1, 6-10 y $15-17$.

47 Palmiter, A. R., Securities regulation, cit., n. ${ }^{\circ}$ 34, 50. 
cosechados por un afiliado del vendedor, debía ser considerada un investment contract, y por ello, un security.

En circunstancias similares, el Noveno Circuito, actuando como Corte de Apelaciones (Court of Appeals) en la jurisdicción federal ${ }^{48}$, consideró también investment contract la venta de unas hormigas que, con pacto de retroventa ${ }^{49}$, serían luego dirigidas el mercado de agropecuario que, por lo general, es el que se beneficia de un producto de esta naturaleza ${ }^{50}$.

De forma semejante, el Quinto Circuito de Estados Unidos, en SEC v. Koscot Interplanetary Inc., calificó la venta de unas participaciones sobre un esquema piramidal como un security, en la medida en que el vendedor reconocía a aquel comprador que trajera otros compradores una suerte de comisión por cada uno de los nuevos clientes adicionales que se adjuntaran por esa vía ${ }^{51}$.

Así, entonces, la idea de los contratos de inversión, como reflejo propio de la jurisprudencia estadounidense, entra a marcar un derrotero de alcance mundial, inclusive frente a las ideas españolas de "valor negociable" o "instrumento financiero", y chilena, peruana, mexicana y colombiana de "valor' 52 , en la medida en que, en nuestros días, Estados Unidos es la principal plaza de negociación de este tipo de instrumentos, y por ello esta noción es la referencia necesaria para los jugadores de este mercado. Y es que, indudablemente, dicho país se muestra en todo el mundo como el principal referente en el mercado de valores, no solo porque constituye el escenario más representativo en esta práctica ${ }^{53}$, sino también porque se erige como una de las plazas más propicias para la innovación, entre otras, a través de la inclusión de nuevos valores por medio de la aplicación de este test.

48 El derecho estadounidense divide su jurisdicción de manera estatal y federal. Conforme a la Judiciary Act de 1789, son 13 los circuitos en los que se dividen las Cortes de Apelación (Courts of Appeals), siendo la novena aquella que tiene jurisdicción sobre California, Arizona, Oregón, Idaho, Montana, Nevada y Washington. Alcaraz, E. et al., El inglés jurídico norteamericano, Barcelona, Ariel, 2016, 61-64.

49 En tal sentido, la decisión del noveno circuito podría basarse en la posible semejanza de la venta mencionada a una operación repo o simultáneas, en la medida en que, por definición, tales transacciones implican, al final, una adquisición con pacto de retroventa. VARÓN PALOMINO, J. C. y ABELLA Abondano, G. D., Régimen del mercado de valores, Intermediación en el mercado de valores, $\mathrm{t}$. III, Bogotá, Ediciones Uniandes-Temis, 2018, 266-267.

50 Smith v., Gross, 604 F.2d. 639, $9^{\text {th }}$ cir. 1979.

51 SEC v., Koscot Interplanetary, Inc., 497 F.2d 473, 1974 U. S. App. Lexis 7641, Fed. Sec. L. Rep., $\mathrm{CCH}, \mathrm{P} 94,710,5^{\text {th }}$ cir. Ga. July $15,1974$.

52 Varón Palomino, J. C., Abella Abondano, G. D., Introducción al derecho del mercado de valores, cit., n. ${ }^{\circ} 1,228-230$.

53 Piénsese, nada más, en la existencia de las plataformas transaccionales más representativas de mercado de valores, como lo son NYSE (hoy NYSE Euronext, por su fusión con la bolsa europea) y NASDAQ, ambas con sede principal en la ciudad de Nueva York. Varón Palomino, J. C., Abella Abondano, G. D., Régimen del mercado de valores. Otras instituciones de los mercados de valores, tomo IV, Bogotá, Ediciones Uniandes-Temis, 2019, 188. 


\section{Test de capital de riesgo (risk capital test)}

Con menos frecuencia que en el supuesto estudiado con anterioridad, el mercado de valores estadounidense, jurisprudencialmente, ha empleado el test de capital de riesgo o risk capital test para evaluar la existencia de un security frente a instrumentos no listados en la norma.

De acuerdo con este test, en aquellos casos en que un inversionista realice un desembolso para inversión, y ésta esté sujeta al riesgo de la empresa sobre la cual el inversionista no tiene control sobre la transacción, habrá un security ${ }^{54}$.

El test, que por demás sirve para determinar si las blue sky laws ${ }^{55}$ de un estado federado son o no aplicables a una transacción concreta, ha visto su manifestación en casos como el conocido por el Segundo Distrito de California, que en Silver Hills Country Club v. Sobieski, y evaluando la naturaleza de las membresías de un club social, consideró que estas configuraban un security en los casos en que los aportes o inversiones realizados por sus miembros fueran destinados a la financiación de las instalaciones y los servicios del club ${ }^{56}$.

\section{Securities en diferentes circunstancias}

Así como la idea de security bien puede encasillarse en cualquiera de los mencionados conceptos, las nuevas realidades de la economía han revelado la necesidad de aplicar la noción en diferentes ámbitos, todos ellos altamente relacionados con la vida cotidiana de los ciudadanos e inversionistas.

Por ello, el régimen del mercado de valores estadounidense, muy consecuente con su tradición práctica, ha venido desarrollando la idea de security en diferentes contextos, esto es, en situaciones diferentes de las que tradicionalmente ocupan un lugar predilecto para su desarrollo.

\section{Real estate como securities}

Una de las primeras manifestaciones novedosas de security en el derecho estadounidense, ya a nuestros días con alguna antigüedad, es la idea del real estate como un security. Bajo esta noción, las operaciones económicas realizadas sobre bienes raíces, en ocasiones, pueden llegar a constituirse como un security.

54 Palmiter, A. R., Securities regulation, cit., n. ${ }^{\circ}$ 34, 51.

55 Llamadas así por los estafadores de la época que prometían abundancia de lluvia en los campos y al final solo dejaban cielos azules, estas normas buscan evitar las estafas que pueden darse por la desregulación de ciertos vendedores de securities. Miller, G. P. y Macey, J. R., "Origin of the Blue Sky Laws", Texas Law Review, vol. 70, n. . 2, rev. 347, 1991, 348-359.

56 Silver Hills Country Club v. Sobieski, 55 Cal. 2d 811, 361 P.2d 906, 13 cal. Rptr 186, 1961. 
Efectivamente, si bien la regla general indica que en principio las operaciones de real estate no se configuran como transacciones de securities, lo cierto es que, aplicando el criterio contenido en Howey - effort of others-, la tradición estadounidense ha admitido la asimilación del real estate como security bajo una circunstancia particular, esto es, que se perciba el esfuerzo de otros mencionado en Howey.

Así, las cortes estadounidenses, identificando la necesidad de dar frente a este nuevo fenómeno, han reconocido el real estate como security en circunstancias como aquella en la que el promotor de un condominio comparte las rentas recibidas por el arrendamiento de las unidades con los demás agentes de la operación. Este reconocimiento vía jurisprudencial, más tarde, provocaría un reflejo en la legislación, que a través de la Securities Act rel. 5347 (1973) se ocupó de establecer las previsiones concretas para indicar en qué casos las ofertas y transacciones realizadas sobre los condominios de tiempo compartido configuran una security ${ }^{57}$.

A partir de allí, el régimen estadounidense se empeñó en reconocer diferentes transacciones inmobiliarias como constitutivas de security. Sobre el particular, dos antecedentes jurisprudenciales sobresalen frente a los demás.

El primero de ellos se presentó cuando un constructor llevó a cabo un proyecto de real estate y vendió los derechos de las unidades del condominio bajo un contrato de ocupación restringida a terceros. Bajo el contrato, el adquirente se hizo a unos derechos restringidos de ocupación, permitiendo a su vez que el constructor o desarrollador arrendara las unidades de las cuales el adquirente era "propietario". Este último recibía pagos por tales arrendamientos del fondo general de rentas que era administrado por el constructor o desarrollador, quien percibía lo pagado por el disfrute de tales unidades a lo largo del tiempo.

El segundo de los casos se presentó ante el juez del Noveno Circuito de los Estados Unidos, quien estableció que en aquellos casos en que un agente inmobiliario ofrece un condominio de renta y ayuda al comprador a celebrar acuerdos con una compañía externa para la administración de la propiedad, hay también una transacción que configura un security ${ }^{58}$. Esta última, que no era más que la venta de un condominio con un acuerdo colateral de administración por parte de un tercero, fue tenida como un security, fundamentalmente porque implicaba la verificación de un esfuerzo de otros en el esquema negocial (Howey $)^{59}$.

Sin embargo, reconociendo la importancia de aclarar las hipótesis en las cuales las transacciones de real estate pueden considerarse un security, el Séptimo Circuito de los Estados Unidos estableció que para que ello ocurra: (1) tiene que haber un fondo de rentas (pooling of rents) del cual se distribuyan las utilidades; y (2) cada

57 Berman, A. y Stone, A., Federal Securities Law and the Sale of Condominiums, Homes, and Homesites, The Business Lawyer, 30(2), 1975, 411-431, disponible en [www.jstor.org/stable/40685379] [consultado el 28 de marzo de 2020].

58 Hocking $v$ Dubois, 885 F.2d 1449, $9^{\text {th }}$ cir. 1989.

59 Securities and Exchange Commission v. W. J. Howey Co., 60 F. Supp. 440, S. D., fla. 1945. 
uno de los propietarios de las unidades del condominio tiene que tener control sobre los contratos que celebra sobre las unidades ${ }^{60}$.

Trazados entonces los principales derroteros para la identificación del real estate como security, el derecho estadounidense presenta este primer antecedente de reconocimiento de nuevos valores, el cual, hoy en día, sirve al mercado como un importante instrumento para la negociación de valores relacionados con bienes raíces en el mercado de capitales no intermediado ${ }^{61}$.

\section{Business interests como securities}

La consideración de una "participación empresarial" o business interest como security guarda una estrecha relación con la naturaleza societaria de la empresa cuya participación se negocia.

Por regla general, y bajo la regulación estatal ${ }^{62}$, se tienen como securities: (1) las acciones ordinarias (common shares) y las preferenciales (preferred shares) en las corporaciones (corporations); (2) las participaciones limitadas de un socio (limited interest partnership) en las compañías limitadas (limited partnership); (3) los intereses de los miembros pasivos (passive member interests) en compañías de responsabilidad limitada (limited liability company [LLC]); así como (4) los intereses de socios pasivos (passive partner interests) en los "contratos de colaboración" (partnershipjoint venture $)^{63}$.

Y no se consideran tal, por su parte: (1) las participaciones generales de un socio (general partner interests) en compañías limitadas; (2) los intereses de miembros activos (active member interests) en las compañías de responsabilidad limitada; y (3) los intereses de socios activos (active partner interests) y de coasociados (co-venturer interests) en los contratos de colaboración empresarial ${ }^{64}$.

60 Wals v. Fox Hill Development Corp., 828 F. Supp. 623, E.D. Wis. 1993

61 BLOOMBERG, Are you a robot?, 12 de noviembre de 2018, en [www.bloomberg.com/tosv2.html?vid= \& uuid=1bf2c180-c9d2-11e9-8673-f177581ea0ba \& url=L25ld3MvdmlkZW9zLzIwMTgtMTEtMTIV YmxvY2tjaGFpbi10YWtlcy1tYW5oYxr0YW4tdmlkZW8=] [consultado el 28 agosto de 2019].

62 Por regulación estatal se hace referencia a aquella que, contrario a la regulación federal, aplica de manera exclusiva en el estado respectivo. Alcaraz Varó, E. Campos Pardillos, M. A. y Miguélez, C., El inglés jurídico norteamericano, 3. a ed., España, Editorial Planeta, 2016, 46-48.

63 En los contratos de colaboración empresarial como los mencionados, suele haber dos tipos de asociados o partners: (1) los operacionales (operational partners) y (2) los pasivos (passive partners). En estos negocios jurídicos de colaboración, las participaciones de los passive partners, quienes por lo general realizan la mayor inversión, bien sea como deuda o como equity, es considerada un valor por regla general. Ello es así, precisamente, porque al igual que ocurre con las participaciones de los general partners en las limited liability partnerships (LLP), su aporte goza de los caracteres propios de los valores, precisamente por el subyacente patrimonial de la participación. En Colombia ello se asemejaría al tratamiento de los socios comanditarios en las sociedades en comandita por acciones, quienes gozan de similar trato.

$64 \mathrm{Al}$ igual que ocurre con las participaciones de los limited partners en las LLP, las participaciones de dichos asociados, debido a la predominancia del factor de administración sobre el de capital en la 
Sin embargo, tales reglas generales en materia estatal, como ocurre en muchas ocasiones, encuentran excepciones una vez pasan por la consideración de las autoridades federales. Esto último aconteció en Williamson v. Tucker, 645 F.2d 404 (5th Cir. 1981), en donde se marcó un importante derrotero sobre la consideración de determinadas participaciones empresariales como securities desde el punto de vista de la jurisprudencia federal.

Sobre el particular, Williamson indicó que en algunas ocasiones las participaciones empresariales podrían considerarse investment contracts. Sobre el particular, estableció que para que ello aconteciera la inversiones realizadas en tales empresas debían presentar, cuando menos, alguna de las siguientes características: (1) ausencia de control legal, por medio de acuerdo como ocurre en las compañías limitadas; (2) incapacidad de control, como en los casos en que, debido al desconocimiento del mercado el socio/miembro, no se tiene ningún manejo sobre la empresa; o (3) inexistencia de control práctico, debido a la dependencia del socio/miembro frente a la habilidad de manejo del administrador ${ }^{65}$.

Tales lineamentos fueron los que, a la postre, establecieron un primer derrotero, conforme al cual las participaciones empresariales pueden ser también consideradas securities a pesar de la regla general estatal, siempre que las funciones de administración sean libradas completamente al administrador. Así, bajo tal presupuesto, se reconocieron como securities, en el caso concreto, las participaciones generales de una compañía limitada.

Con posterioridad, el Cuarto Circuito de los Estados Unidos dio a conocer su posición propia, e indicó que solamente las inversiones en las que hay ausencia de control legal del inversionista podían llegar a ser consideradas contratos de inversión, y, por ende, valores ${ }^{66}$.

Sin embargo, una precisión no dejaba de causar dudas en el grueso del ejercicio de la abogacía y la judicatura, y es ¿en qué momento se debe evaluar ese control? Frente a ello, el pronunciamiento del Decimoprimer Circuito de Estados Unidos en SEC v. Merchant Capital, LLC, 483 F.3d 747 (11th Cir. 2007) prestó un gran servicio, al mencionar que, en cualquier caso, la evaluación del control debía hacerse al momento de la inversión, de manera que sería este el tiempo para determinar la existencia del security bajo estas reglas.

Por su parte, las empresas unipersonales (one-person LLC) y las co-owned LLC plantean una consideración particular: las primeras, por no configurar securities debido a la ausencia de inversiones externas; y las segundas, por permitir que sus par-

asociación, dichas participaciones no suelen ser consideradas un valor, en parte porque en ellas se pueden percibir rasgos de lo que sería una participación en una sociedad de carácter personal. En Colombia dicho tratamiento se asemeja al dado a los socios gestores en las sociedades en comandita por acciones.

65 Williamson v. Tucker, 645 F.2d 404, $5^{\text {th }}$ cir. 1981.

66 Rivanna Trawlers v. Thompson Trawlers, 650 F. Supp. 1378, W. D. Va. 1986. 
ticipaciones sean consideradas securities con base en el criterio del esfuerzo de otros y las expectativas del inversionista ${ }^{67}$ ya referidas.

Así, si bien el desarrollo jurisprudencial a este punto es importante, y establece unos criterios fundamentales para la determinación de la existencia de un security en diferentes contextos, lo cierto es que hoy en día, si bien no se cuenta con un criterio rígido o estricto que permita saber cuándo las participaciones empresariales son securities, sí se percibe un parámetro singular para analizar en cada caso la existencia de aquellos de forma concreta ${ }^{68}$.

\section{Planes pensionales como securities}

Los planes pensionales, como valores, se restringen a un ámbito muy concreto de la regulación pensional, y es el propio de los planes que son administrados por los fondos de trabajadores. En efecto, contrario a lo que ocurre por regla general en las tradiciones romano-germánicas, los regímenes anglosajones, y particularmente el estadounidense, permiten la constitución y administración de fondos pensionales por parte de los trabajadores ${ }^{69}$.

Tales fondos, importantes jugadores del sistema económico, dieron lugar a que la Corte Suprema de Justicia de Estados Unidos estableciera un criterio claro para evaluar si las inversiones en un fondo pensional pueden o no ser consideradas securities. Tal criterio, que toma como base la renta que se percibe de ellos, establece que si las inversiones se realizan bajo un esquema de "renta variable" (variable-benefit), habrá un security, mientras que si se otorga bajo un esquema de renta fija (fixed-benefit), no se verificará la existencia de tal instrumento ${ }^{70}$.

Sin embargo, la claridad de tal criterio, el cual no es otra cosa que la lectura aplicada al caso concreto de Howey, encuentra un obstáculo cuando se analiza la aplicación de la Employee Retirement and Income Security Act (ERISA). La discusión sobre el particular, cada día menos reiterada, presenta dos posturas, esto es, de quienes consideran que la aplicación del ERISA excluye cualquier regulación de valores

67 Palmiter, A. R., Securities regulation, cit., n. ${ }^{\text {o } 34,54-55 . ~}$

68 Las realidades económicas han planteado la discusión sobre si las inversiones en los esquemas Ponzi o pirámides pueden ser consideradas securities. Sobre el particular, el Primer Circuito de los Estados

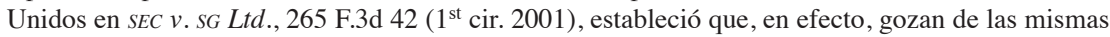
características de aquellos, especialmente porque: (1) sirven para atraer inversionistas, (2) tienen un administrador con amplios poderes de administración, (3) se otorgan bajo una promesa de retornos económicos futuros y (4) cuentan con un administrador quien realiza con sus esfuerzos las labores para recibir réditos económicos. Por ello la SEC se ha valido de la consideración de tales inversiones como securities, en específico, para indicar que, al revestir tal calidad, y no encontrarse registrados, la autoridad podría adelantar acciones de enjuiciamiento civil en su contra.

69 La principal norma que regula la constitución de fondos de pensiones está en la Employee Retirement Income Security Act of 1974 (ERISA), contenida en el capítulo 18 de la parte 29 del U. s. C.

70 Teamsters v. Daniel, 439 U. S., 551, 1979. 
aplicable a tales fondos, y aquella de quienes, como la SEC, consideran que la regulación de valores se aplica independientemente de regulación del ERISA.

Otra discusión, igual de importante, y parcialmente aclarada en 1998 por el Circuito Decimoprimero de los Estados Unidos, va sobre la aplicación de los instrumentos otorgados bajo el modelo Stock Appreciation Rights (SAR $)^{71}$. Sobre el particular, la autoridad ${ }^{72}$ consideró que dichos instrumentos no son securities por dos motivos: (1) porque no son transferibles en ninguna circunstancia; y (2) porque no otorgan a sus tenedores derechos sobre la propiedad de la empresa. Sin embargo, la discusión revivió un año más tarde, pues la decisión mencionada fue revocada en $1999^{[73]}$ debido a la falta de legitimidad por activa del demandante ${ }^{74}$.

De manera que, en la actualidad, así como acontece en algunas de las situaciones indicadas previamente, los instrumentos mencionados gozan de la calidad de security, en particular, siguiendo la línea de Howey y los contratos de inversión ${ }^{75}$. Así, no siendo los planes pensionales la excepción, el reconocimiento de estos como securities muestra una realidad ineludible, cual es que cada vez más instrumentos no tradicionales del mercado financiero entran en éste a participar como mecanismos de negociación.

\section{Notas como securities}

En el derecho estadounidense las notas ocupan un lugar intermedio entre los papeles comerciales y los bonos, en específico porque, si bien al igual que estos son títulos de deuda a cuyo vencimiento se puede solicitar el valor pagado (con los intereses pactados), las notas tienen un periodo de vencimiento mucho menor que el de aquellos, pero superior al de los papeles comerciales ${ }^{76}$.

71 Se trata de una forma de compensación para los trabajadores similar a las opciones otorgadas a estos sobre acciones. En concreto, este tipo de compensación consiste en reconocer al trabajador que lo tiene, el valor en efectivo del incremento de la acción en un periodo determinado. Se conceden en unidades y con periodos de maduración determinados. Por ejemplo, si a un empleado se le conceden 100 SAR, con un periodo de maduración de 5 años, y en el transcurso de ese tiempo la acción incrementó, por ejemplo, uSD 50, el valor de la compensación será igual a \$5000 uSD (se deriva de la siguiente operación: 100 unidades x usD 50). Si se llegaren a reconocer como dinero utilizable para ejercer una opción sobre acciones, se denominarían Tandemsar.

72 Clay v. Riverwood International Corporation, 157 F.3d 1259, $11^{\text {th }}$ cir. 1998.

73 Reh'g 176 F.3d 1381, $11^{\text {th }}$ cir. 1999.

74 Palmiter, A. R., Securities regulation, cit., en n. ${ }^{\circ}$ 34, 56-57.

75 Sobre el particular, el Noveno Circuito de los Estados Unidos recuerda que los regalos de caridad (charitable gift annuities) pueden ser considerados contratos de inversión y sin embargo exceptuados del registro ante la SEC. Warfield v. Alaniz, 569 F. 3d 1015, $9^{\text {th }}$ cir. 2009.

76 Los bonos son instrumentos de deuda con largos periodos de maduración, por lo general, superiores a un año (los bonos del Tesoro de Estados Unidos maduran de dos a diez años). Las notas también son instrumentos de deuda, pero con un periodo de maduración que suele variar entre uno y cinco años, o inclusive, inferior a un año. Los papeles comerciales, a su vez, son igualmente títulos de 
Bajo la regulación estadounidense, las notas, como los bonos, son por regla general securities. En efecto, la naturaleza propia de tal tipo de instrumento de deuda muestra con claridad por qué tanto la Securities Act como la Securities Exchange Act tienen a las notas como valores por antonomasia.

Sin embargo, como se indicó en su momento, el derecho angloamericano contiene una cláusula de contexto para los casos en que, existiendo un instrumento que a priori debe tenerse como security, no puede considerarse tal bajo el contexto particular. Tal es el caso de las notas que, no pudiendo eludir la pertinente provisión del legislador, pierden su naturaleza de valor en determinadas circunstancias concretas.

Sobre la naturaleza de estas como securities, la conclusión se ha orientado a decir que (1) las notas que se usan para operaciones comerciales y de consumo en general no son securities, mientras que (2) las notas vendidas a inversionistas en emisiones que se comercian con la promesa de una retribución económica atada al monto de la inversión sí son tenidas como tal ${ }^{77}$.

Así, si bien no son menos aplicables las pruebas Howey y similares, la Corte Suprema de Justicia ha determinado que, salvo que las notas puedan encasillarse dentro de instrumentos que por su contexto no deban ser securities, la regla general indica que deben tenerse como tal, esto, de conformidad con lo señalado por la Corte Suprema en Family Resemblance Test en Reves v. Ernst \& Young ${ }^{78}$.

En dicha providencia, además de lo mencionado se estableció que son varios los criterios que ha de tener en cuenta el juez al momento de la evaluación de una nota como security. En concreto, debe analizarse: (1) la motivación del comprador/ vendedor; (2) el plan de distribución; (3) las expectativas razonables del público inversionista; y (4) otros factores que, en su conjunto, permitan saber si se trata de un valor o $\mathrm{no}^{79}$.

\section{Sale-leaseback financing}

Los sale-leasebacks son instrumentos financieros por medio de los cuales un financiador compra un activo para que una persona, en ocasiones ajena a esa compraventa, disfrute del bien a cambio del pago de unas sumas periódicas, y así también de una suma final que hace las veces de pago del préstamo inicial ${ }^{80}$.

deuda con un periodo de maduración que, por ser igual o inferior a nueve meses, los excluye de la regulación del mercado de valores en Estados Unidos.

77 Eso es así, precisamente porque mientras las primeras responden primordialmente a una necesidad de consumo, en la que no hay una inescindible intención de inversión, las segundas sí reconocen tal naturaleza, pues en lugar de constituir un mero instrumento de pago (como lo es el pagaré), figuran como un mecanismo de inversión. PALMiter, A. R., Securities regulation, cit., n. ${ }^{\circ}$ 34, 57-59.

78 Reves v. Ernst \& Young, 494 U. S., 56, 1990.

79 Idem.

80 Palmiter, A. R., Securities regulation, cit., n. ${ }^{\circ}$ 34, 59. 
La naturaleza de ese instrumento, muy ligada a la noción de 'arrendamiento', no se tiene por lo general por un security, salvo en aquellos casos en que no siendo el acreedor un prestamista comercial sino un grupo de especuladores, se realice la operación mencionada ${ }^{81}$.

En tales circunstancias, lo que en principio se tiene como un préstamo garantizado $^{82}$, precisamente por la conservación de la titularidad del activo por parte del acreedor, se convierte en un security, concretamente porque el respectivo instrumento financiero se erige como un contrato de inversión. Así lo consideró la Corte Suprema de los Estados Unidos en SEC v. Edwards ${ }^{83}$, cuando evaluando los particulares del caso dijo: "An investment scheme promising a fixed rate of return can be an 'investment contract' and thus a 'security' subject to the federal securities laws" 84 . En efecto, tal premisa fue la que, en este caso, abrió la posibilidad de que, dentro de determinados derroteros, las operaciones de leasing se consideraran securities.

\section{Instrumentos bancarios como securities}

Con razón, señala Palmiter, las cuentas de ahorro y los certificados de depósito son instrumentos financieros considerados valor por antonomasia ${ }^{85}$. Sin perjuicio de ello, la aplicación de la "cláusula de contexto" mencionada permitió que en el caso Marine Bank v. Weaver, 455 U. S. 551 (1982) los certificados de depósito expedidos por bancos nacionales no fueran tenidos como securities, sugiriendo implícitamente que la suficiencia de la regulación bancaria federal sobre tales instrumentos hacía innecesaria la aplicación de la regulación del mercado de valores ${ }^{86}$, pues con esta primera bastaba para satisfacer las necesidades propias de estas actividades.

\section{La regla general de la finalidad como criterio para identificar un security}

Sumado a todo lo anterior, y como respuesta evidente a las necesidades de la economía en esta área particular del derecho, se debe reconocer que el derecho estadouni-

81 Cuando se trata de un grupo de especuladores, a la operación le subyace una clara intención de inversión, por lo cual, se pueden llegar a tener dichos contratos como securities.

82 En Colombia la utilización de operaciones de repo, simultáneas y compraventas con reserva de dominio cumplen funciones similares a lo que se verifica en este caso. Inclusive, debido a la funcionalidad que se deriva de la Ley de Garantías Mobiliarias Colombiana (Ley 1676 de 2013). Así mismo, llegaría a ser una garantía admisible en los términos del artículo 3 del Decreto 2360 de 1993, y una garantía idónea en los términos del capítulo in de la Circular Externa 100 de 1995 -Circular Básica Contable y Financiera.

83 SEC v. Edwards, 540 U. S., 2004, 389.

84 "Un esquema de inversión en el cual se promete una tasa fija de retorno puede ser un contrato de inversión y por ello un valor sujeto a las leyes federales."

85 Palmiter, A. R., Securities regulation, n. ${ }^{\circ} 34,60$.

86 Ibid., 61. 
dense ha dotado de un papel fundamental a la realidad económica de la operación al momento de considerar security a un instrumento financiero.

En efecto, el factor común en la regulación angloamericana estudiada es el reconocimiento de los securities, más que por su nombre o denominación, por la intención y el objeto económico que les subyace. De ahí que sea esta, a más de las anteriores, la realidad vertical que se deba reconocer al identificar un security ${ }^{87}$.

\section{Implicaciones de denominar un instrumento security}

Las implicaciones de denominar security a un determinado instrumento, tanto en el régimen estadounidense como en el colombiano, son principalmente dos, a saber: (1) la posibilidad de acceder a un mercado de financiación en buena medida provechoso para el emisor; y (2) la necesidad de sujetarse a un riguroso régimen jurídico derivado de este particular.

En efecto, la primera de las implicaciones de denominar 'valor' a un instrumento presenta una ventaja, cual es que al ser un mecanismo de captación de recursos originado para la realización de financiación "des-intermediaria”, permite al emisor tener acceso a un mercado muchas veces más provechoso que aquel al cual, sin ser valor, podría tener acceso. Dicha realidad presenta una ventaja mayúscula para la realización de financiación, en especial porque permite al demandante de recursos encontrar un mercado con agentes mucho más calificados y dispuestos a realizar la inversión requerida.

Sin embargo, tal ventaja tiene a su vez una contrapartida, que en este caso lleva al segundo punto anunciado, cual es la necesidad de cumplir con un régimen jurídico estricto en materia del mercado de valores.

Dicho régimen, encaminado fundamentalmente a la morigeración de las falencias en materia de información, y así también, a aquello que recoge la Organización Internacional de Comisiones de Valores (IOSCO, por su sigla en inglés [International Organization of Securities Commissions]) en sus objetivos principales, a la consecución de los objetivos de: (1) protección de los inversionistas; (2) justicia, eficiencia y transparencia en los mercados; y (3) reducción del riesgo sistémico ${ }^{88}$, implica que, en su respectiva medida, todos los agentes o individuos que acudan al mercado de valores se sujeten a una regulación específica sobre este.

De ahí que si bien se puede encontrar un enorme beneficio en la financiación por medio del mercado de valores, lo cierto es que tal acceso implica la observancia de

87 Una frase pronunciada por la Corte Suprema de Estados Unidos ilustra con claridad esta circunstancia: "[S]ecurities transactions are economic in character Congress intended the application of these statutes to turn on the economic realities underlying a transaction, and not on the name appended thereto". United Housing Foundation, Inc. v. Forman, 421 U. S., 1975, 837.

88 Varón Palomino, J. C. y Abella Abondano, G. D., Introducción al derecho del mercado de valores, cit., n. ${ }^{\circ} 1,4$. 
una regulación rigurosa que, bajo la consideración de un determinado instrumento como valor, establece el cuidadoso cumplimiento de la totalidad de las normas del mercado de valores por quien acude al respectivo escenario.

\section{Aportes de la regulación de valores en Estados Unidos al régimen jurídico colombiano}

Acotadas las consideraciones anteriores, creemos útil realizar algunas reflexiones sobre las posibles lecciones que pueden aprenderse de la experiencia estadounidense, las cuales, en su mayoría, se relacionan con la forma en que se lleva a cabo el acercamiento al concepto de 'valor'. Se recuerda que, por la extensión misma del presente estudio, las consideraciones expuestas en el presente acápite pretenden, más que dar soluciones irrestrictas sobre los temas que se plantean en el presente documento, elevar interrogantes que puedan llegar a tener un impacto en la manera en que se lleva a cabo el acercamiento al concepto de 'valor' en el régimen jurídico colombiano.

Así, lo primero, será preguntarse si es o no conveniente tener una noción amplia de 'valor' en el régimen jurídico colombiano. En nuestro sentir, la conveniencia de dicha previsión se revela más que evidente en nuestros días, en especial, debido a la dinámica propia de movimiento que se percibe en el mercado de valores, tanto en el mercado principal como en el segundo mercado ${ }^{89}$. Sobre ello, aun cuando creemos que el artículo 2. ${ }^{\circ}$ de la Ley 964 de 2005 ya incluye una previsión amplia de dicho concepto, la aplicación de esta se ha visto entorpecida como consecuencia de la forma en que la autoridad ha realizado la interpretación de la norma.

En efecto, creemos que, desde el punto de vista del ejercicio comparado, se pueden tomar, en el derecho colombiano, varios de los elementos que se tienen en cuenta para realizar el acercamiento al concepto de 'valor' en Estados Unidos. Los más destacables, a nuestro juicio, tienen que ver con la verdadera aplicación efectiva de un concepto amplio de 'valor', cuya valoración se lleva a cabo en dos momentos con especial relieve, esto es: (1) cuando el abogado que asiste la emisión califica un documento o instrumento como tal para efectos de la aplicación del respectivo régimen; y (2) cuando el juez de conocimiento lleva a cabo la calificación definitiva de 'valor' ante una controversia" ${ }^{90}$.

Así mismo, creemos del caso interrogarnos si bajo el régimen colombiano puede eventualmente incluirse una disposición en el sentido de privar de la calidad de valor

89 Recientemente, la Misión del Mercado de Capitales, dentro de sus recomendaciones emitidas en el segundo semestre del 2019, sugirió la modificación de dichas rúbricas por las de "mercado público" y "mercado privado". Disponible para consulta en [www.urf.gov.co/webcenter/portal/urf/pages_c/ misinmercadodecapitales] [consultado el 28 de marzo de 2020].

90 Por la mecánica que es propia en dicha regulación, la evaluación se lleva a cabo en esos dos momentos. Esto permite, fundamentalmente, que no se entorpezca la mecánica del mercado y de la economía. En particular, creemos que la adopción de un esquema similar no solo permite que la economía se mueva de una forma más dinámica, sino que también da lugar a la innovación. 
a aquellos instrumentos que por antonomasia se consideran tal, como ocurre, por ejemplo, en el caso estadounidense con la aplicación de la "cláusula de contexto"91.

En segundo lugar, una evaluación sobre los tipos de valores no tradicionales reconocidos por las autoridades estadounidenses muestra una reflexión interesante, en especial cuando se tienen en cuenta los nuevos retos que los mercados imponen constantemente. En efecto, tales exigencias se muestran claras si se observa, por ejemplo, lo que acontece con los tokens; la titularización en mercado inmobiliario -inclusive a través de la "tokenización" "92_; las membresías de los clubes sociales; los títulos representativos de bienes o deuda inmobiliaria; los instrumentos bancarios; los planes pensionales; así como con todos los demás valores que paulatinamente han llegado a irrumpir la mecánica tradicional del mercado de valores.

Sobre ello, creemos importante reconocer la función del abogado y del juez durante el proceso de calificación de un valor, más inclusive que la del supervisor, pues consideramos que la predominancia del papel de dichos agentes dota de facilidad y dinamismo al mercado de capitales, en especial en el contexto del mercado de valores.

Un tercer aspecto que interesa destacar, en lo que respecta a las reflexiones sobre el concepto de 'valor' en derecho estadounidense frente al derecho colombiano, es aquel que toca con el papel de los actores del mercado de valores en Colombia. Dicho papel, que por demás ya fue cuestionado por la Misión del Mercado de Capitales Colombiana en el 2019, toca las fibras más sensibles de la estructura actual del mercado de capitales en Colombia, sus agentes y regulación.

Sobre el particular, precisamente porque consideramos que su investigación merece un acercamiento más estricto y extenso, nos limitaremos a poner de presente una realidad, cual es que, a diferencia de lo que ocurre en el régimen colombiano, la regulación de valor y su reconocimiento en Estados Unidos hacen que el papel del juez sea más valioso que el del supervisor.

En ese sentido, aunque no descartamos en lo más mínimo la importancia del papel del supervisor, creemos que la práctica ha demostrado que su función, en lo que tiene con el reconocimiento de valores no incluidos en la definición legal, obstaculiza el dinamismo del mercado de valores, e inclusive presenta alguna dificultad para la utilización de instrumentos cuya naturaleza no ha sido rigurosamente definida, como ocurre, por ejemplo, con el Simple Agreement for Future Equity (SAFE) o el Keep It Simple Security (KISS), que hoy se erigen como instrumentos predilectos por determinados inversionistas ${ }^{93}$.

91 Aplicar una cláusula de este tipo en todos los casos resulta clave en el derecho colombiano. Particularmente, a permitir la exclusión de determinados instrumentos como valores, se permite que estos puedan circular sin el cumplimiento del riguroso régimen del mercado de valores, por ejemplo cuando al instrumento no le subyace una finalidad de inversión, sino de consumo.

92 BloOMBerg, Are you a robot?, cit.

93 Los SAFE son valores convertibles creados por Y Combinator. Con ellos, el inversionista realiza un aporte a un proyecto (usualmente un start-up), el cual se convertirá en equity (i.e. acciones), en un momento posterior, concretamente, cuando se hagan las rondas en las que se determinan los niveles 
Y es que consideramos que en la práctica extranjera mencionada, a más de lo dicho frente a la consideración de 'valor' como tal, se puede destacar el papel de otros agentes, que en el derecho colombiano parecen no tener un papel tan protagónico, precisamente por la cooptación de funciones por parte de la rama ejecutiva del poder público. En ese sentido, se muestra interesante ver cómo, siendo el juez el estadio excepcional frente a la duda y la controversia, el abogado desempeña un papel crucial en la evaluación de un valor, en específico, al momento de conceptuar sobre su naturaleza para efectos de una emisión ${ }^{94}$.

Dicho papel, en lo que creeríamos que constituye una práctica saludable para promover la profundización del mercado colombiano ${ }^{95}$, consideramos debe ser un punto que llame la atención y que, cuando menos, muestre el beneficio de rezagar en alguna medida el papel del supervisor sobre este particular. Esto, sin embargo, no es tarea fácil, y requiere, como se advertía en $2019^{[96]}$, la reformulación de algunas de las competencias asignadas a las autoridades en Colombia.

Acotado lo anterior, consideramos que, como ha ocurrido otras veces en la práctica del mercado de valores colombiano, el régimen jurídico de este país puede aprender varias lecciones sobre las experiencias internacionales, especialmente, teniendo presente el contexto actual, que, como lo advierte la doctrina mencionada, y así también la misma Misión del Mercado de Capitales, demanda una modificación en dinámica tradicional del mercado de valores colombiano.

\section{Conclusiones}

Habiendo realizado el acercamiento al concepto de 'valor' desde la regulación colombiana y estadounidense, en lo que tiene que ver con el objeto de esta investigación, se puede concluir:

de participación (no es una nota convertible, en la medida en que no tiene una fecha de maduración ni una tasa de interés atada al mismo). Pueden presentarse de las siguientes formas: (1) valuation cap, no discount; (2) discount, no valuation cap; (3) valuation cap and discount; y (4) MFN, no valuation cap, no discount, dependiendo de si hay un límite de ese dinero que se pueda convertir a equity, y de si la conversión lleva atada un descuento. Los KIss, por su parte, son también valores convertibles, creados por 500 Startups. Estos, pueden presentarse de dos maneras: (1) como KISs equity, en donde se hace una inversión, se tiene el valor que va a ser convertido, pero no se tiene ni tasa de interés ni periodo de maduración por su tenencia; y (2) KIss debt, que tienen una tasa de interés del 5\%, y un periodo de maduración de 18 meses, luego de los cuales se convierten en equity (acciones).

94 Este, en concreto, es un reto que tiene el mercado colombiano, pues parecería conflictuar con las facultades que tiene el Ministerio de Hacienda y Crédito Público y la Superintendencia Financiera de Colombia. De ahí que se ponga de presente, en el estudio, el beneficio de mercado que supone hacer un control como el estadounidense, por encima de uno como el colombiano.

95 Sobre el particular, véanse las Recomendaciones de la Misión del Mercado de Capitales emitida en agosto de 2019 .

96 Aunque en un sentido diferente al formulado, la Misión del Mercado de Capitales reconoció la existencia de vacíos, ambigüedades y duplicidades en la regulación del papel de las autoridades financieras en Colombia. Véanse las Recomendaciones de la Misión del Mercado de Capitales emitidas en agosto de 2019. 
- Primero, que la experiencia estadounidense, a pesar de contar con una regulación estricta en muchos de sus particulares, dota al mercado de valores de una noción amplia de security, a partir de la cual se abre paso a la dinamización y profundización del mercado de valores;

- Segundo, que el régimen estadounidense, a pesar de contar con un listado amplio de documentos que prima facie son considerados valores, contrarresta los efectos indeseados que de ello pudieren derivarse, con la aplicación de dos nociones fundamentales, esto es, la de los contratos de inversión y la de la cláusula de contexto;

- Tercero, que el régimen del mercado de valores colombiano, en principio partícipe de la intención de dinamización a través de la inclusión de un concepto amplio de 'valor', hoy encuentra un fuerte obstáculo para la innovación y el reconocimiento de nuevos instrumentos financieros como valores, precisamente por la restricción que a través de la rama ejecutiva del poder público se ha impuesto a ello;

- Cuarto, que la delimitación y el encauce de los papeles de los agentes del mercado, según la finalidad del mercado de valores, es fundamental en la obtención y asimilación de un concepto amplio de 'valor', que permita un mercado más amplio, diversificado y profundo en Colombia; y

- Quinto, que el mercado y la regulación colombianos en materia de valores pueden sacar provecho de experiencias como la estadounidense, en específico, para abrir la posibilidad de dotar de la calidad de valores a instrumentos financieros novedosos que, siendo muchas veces disruptivos, permiten el acceso para la financiación a nuevos actores del mercado.

\section{Referencias}

\section{Doctrina}

Alcaraz Varó, E.; Campos Pardillos, M. A. y Miguélez, C., El inglés jurídico norteamericano, 3. ${ }^{a}$ ed., España, Editorial Planeta. 2016.

Alcaraz, E. et al., El inglés jurídico norteamericano, Barcelona, Ariel, 2016.

López Roca, L. F., Baquero Herrera, M. H. y Corredor Higuera, J. A., Estudios jurídicos sobre el mercado de valores, primera edición, Bogotá, Colombia.

Berman, A. y Stone, A., Federal Securities Law and the Sale of Condominiums, Homes, and Homesites, The Business Lawyer, 30(2), 1975.

Blanco Barón, C. y Baquero Herrera, M., Cinco años de la Ley 964 de 2005: ¿se están cumpliendo sus objetivos?, Bogotá, Universidad Externado de Colombia, 2011. 
Loss, L. y Seligman, J., Fundamentals of securities regulation, 5. ed., United States of America, Wolters Kluwer, 2004.

Merryman, J.H., The Civil Law Tradition: An Introduction to the Legal Systems of Europe and Latin America, 3. . ed., Stanford, United States of America, Stanford University Press, 2007.

Miller, G. P. y Macey, J. R., "Origin of the Blue Sky Laws”, Texas Law Review, vol. 70, n. 2 , rev. 347, 1991.

Palmiter, A. R., Securities regulation, 7. a ed., New York, United States of America, Wolters Kluwer, 2017.

Steinberg, M. I. y Kaulbach, W. E., "The Supreme Court and the Definition of Security: The Context Clause, Investment Contract Analysis, and Their Ramifications", Vanderbilt Law Review, vol. 40, n. . 3, 1987.

Varón Palomino, J. C. y Abella Abondano, G. D., Derechos fiduciarios y mercado de valores: reflexiones frente a la normativa colombiana, Bogotá, Ediciones Uniandes-Asociación de Fiduciarias, 2013.

Varón Palomino, J. C. y Abella Abondano, G. D., Régimen del mercado de valores. Introducción al derecho del mercado de valores, tomo I, Bogotá, Ediciones Uniandes-Temis, 2017.

Varón Palomino, J. C. y Abella Abondano, G. D., Régimen del mercado de valores. Intermediación en el mercado de valores, tomo III, Bogotá, Ediciones Uniandes, Temis, 2018.

Varón Palomino, J. C. y Abella Abondano, G. D., Régimen del mercado de valores. Otras instituciones de los mercados de valores, tomo IV, Bogotá, Ediciones Uniandes-Temis, 2019.

\section{Jurisprudencia}

\section{Estados Unidos}

SEC v. W.J. Howey Co., 328 U. S. 293, 1946.

Silver Hills Country Club v. Sobieski, 55 Cal. 2d 811, 1961.

SEC v. Koscot Interplanetary Inc., 497 F. 2d 473, $5^{\text {th }}$ cir., 1974. 
Smith v. Gross, 604 F.2d. 639, $9^{\text {th }}$ cir., 1979.

International Brotherhood of Teamsters v. Daniel, 439 U. S. 551, 1979.

Williamson v. Tucker, 645 F.2d 404, 5th Cir., 1981.

Marine Bank v. Weaver, 455 U. S., 551, 1982.

Wartzman v. Hightower Productions, ltd., 53 Md. App. 656, 1983.

Rivanna Trawlers Unlimited v. Thompson Trawlers, Inc., 840 F.2d 236, $4^{\text {th }}$ cir., 1988.

Hocking v. Dubois, 885 F. 2d. 1449, 9th Cir., 1989.

Clay v. Riverwood Int'l. Corp., 157 F. 3d 1259, $11^{\text {th }}$ cir., 1998, y reh'g 176 F.3d $1381,11^{\text {th }}$ cir., 1999.

Wals v. Fox Hills Development Corp., 24 F.3d 1016, $7^{\text {th }}$ cir., 1994.

SEC v. SG Ltd., 265 F.3d 42, $1^{\text {st }}$ cir., 2001.

SEC v. Edwards, 540 U. S. 389, 2004.

SEC v. Merchant Capital, LLC, 483 F.3d 747, 11 $11^{\text {th }}$ cir., 2007.

Warfield v. Alaniz, 569 F. 3d 1015, $9^{\text {th }}$ cir., 2009.

\section{Legislación nacional y extranjera}

\section{Colombia}

Código Civil.

Ley 32 de 1979.

Ley 35 de 1993.

Ley 964 de 2005.

Ley 1676 de 2013.

Código de Comercio. 
Decreto 2969 de 1960.

Decreto 2360 de 1993.

Decreto 2555 de 2010.

Superintendencia Financiera de Colombia, Circular Externa 100 de 1995.

Superintendencia Financiera de Colombia, Concepto 20056-946 del 27 de julio de 2005.

Superintendencia Financiera de Colombia, Concepto 2016054365-015 del 28 de julio de 2016.

\section{Estados Unidos de América}

Securities Act de 1933

Securities Exchange Act de 1934

Securities Act Rel. n. ${ }^{\circ}$ 5347,1973.

Employee Retirement and Income Security Act (ERISA)

\section{Fuentes electrónicas: internet}

BLOOMBERG, Are you a robot?, 2018, noviembre 12, disponible en [www.bloomberg. com/tosv2 html?vid=\&uuid=1bf2c180-c9d2-11e9-8673-f177581ea0ba\&url=L 25ld3MvdmlkZW9zLzIwMTgtmTEtмtivYmxvY2tjaGFpbi10YWtlcy1tYW5 oYXr0YW4tdmlkZW8=] [consultado el 28 de agosto de 2019].

Real Academia de la Lengua Española, disponible en [https://dle.rae.es/srv/fetch? $\mathrm{id}=\mathrm{EjQrABh}]$.

Recomendaciones de la Misión del Mercado de Capitales, disponible en [www.urf. gov.co/webcenter/portal/urf/pages_c/misinmercadodecapitales]. 